SFB

Parameter estimation for the drift of a time-inhomogeneous jump diffusion process

823

Brice Franke, Thomas Kott

Nr. 37/2010

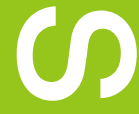



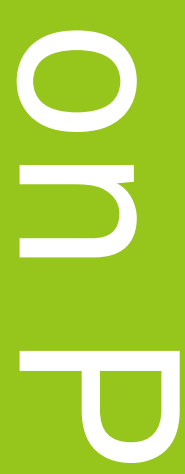

(1)

(D)

D

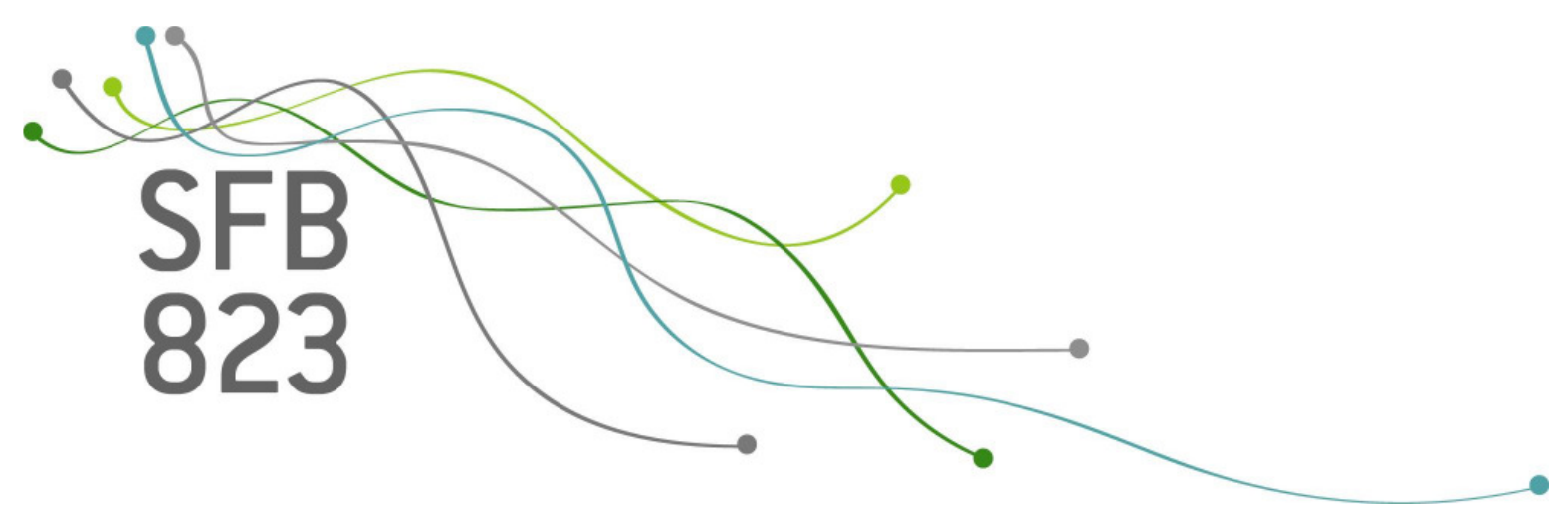



August 30, 2010

\title{
PARAMETER ESTIMATION FOR THE DRIFT OF A TIME-INHOMOGENEOUS JUMP DIFFUSION PROCESS
}

\author{
BRICE FRANKE AND THOMAS KOTT
}

\begin{abstract}
This work deals with parameter estimation for the drift of jump diffusion processes which are driven by a Lévy process and whose drift term is linear in the parameter. In contrast to the commonly used maximum likelihood estimator, our proposed estimator has the practical advantage that its calculation does not require the evaluation of the continuous part of the sample path. In the important case of an Ornstein-Uhlenbeck-type jump diffusion, which is a widely used model, we prove consistency of our estimator.
\end{abstract}

\section{INTRODUCTION}

In statistical inference for time-continuous stochastic processes, parameter estimators that are based on the observation of the entire time-continuous path are natural objects to study: These estimators have often a closed-form representation in terms of stochastic integrals such that large sample results like consistency and asymptotic normality may be obtained by using techniques from stochastic analysis, see Section 5. In many situations continuous time estimators can be fairly approximated by their discrete time versions, see Lemma 3.1 for a representation.

There exists a large number of publications on drift parameter estimation for timecontinuously observed diffusion processes. Maximum likelihood estimation is thereby, as well as in many other fields of statistical inference, the most commonly used estimation method. For a continuous diffusion process, which is an important model in many applied fields, with stochastic differential

$$
d X_{t}=f\left(t, X_{t}, \theta\right) d t+d B_{t}, \quad 0 \leq t \leq T,
$$

where $\left(B_{t}\right)_{t \geq 0}$ denotes Brownian motion and $\theta$ the unknown parameter, maximum likelihood estimation is based on Girsanov's theorem which provides an expression of the likelihood function. The resulting maximum likelihood estimator requires the computation of integrals of the form

$$
\int_{0}^{T} f\left(t, X_{t}, \theta\right) d X_{t}
$$

Asymptotic properties of maximum likelihood estimates from time-continuous realizations of the process in (1) can be found e.g. in [3] and [15]. Given time-continuous observations $\left\{X_{t}, 0 \leq t \leq T\right\}$ the integral in (2) is approximated by an Itô sum using time-discrete increments of the sample path.

Especially in mathematical finance, an important generalization of model (1) is the jump diffusion process allowing for the possibility of discontinuities and a wide variety of marginal

Key words and phrases. Time-inhomogeneous diffusion process, time-continuous sample, least squares estimation, maximum likelihood

Acknowledgement. This work was partly supported by the Collaborative Research Center 'Statistical modeling of non-linear dynamic processes' (SFB 823) of the German Research Foundation. 
distributions, see [2] and [6] for some applications. The jump diffusion process is defined as the solution to

$$
d X_{t}=f\left(t, X_{t}, \theta\right) d t+d L_{t}, \quad 0 \leq t \leq T,
$$

where $\left(L_{t}\right)_{t \geq 0}$ is a homogeneous Lévy process, and the application of the maximum likelihood approach to the Girsanov density yields estimators that are based on

$$
\int_{0}^{T} f\left(t, X_{t}, \theta\right) d X_{t}^{c}
$$

where $X_{t}^{c}$ is the continuous part of the process. This integral cannot be computed without further ado since the continuous part is not observed separately in practice. Large sample results on the maximum likelihood estimator for jump diffusion processes are derived in [19] and $[20]$.

In this treatment we present an alternative continuous-time estimator for the drift parameter $\theta$ of the jump diffusion process given in (3) where the drift term is linear in the parameter, that means that the process solves

$$
d X_{t}=f\left(t, X_{t}\right) \theta d t+d L_{t}
$$

Our estimator is derived by making use of the least squares method. In detail, we first regard the discretized version of the stochastic differential equation (4) and apply ordinary least squares estimation. In doing so, we obtain a time-discrete estimator. Thereafter, we take the limit as the discretization step $\Delta t$ goes toward zero and get thereby a continuous time estimator of the drift parameter. Note that the resulting least squares estimator does not coincide with the trajectory fitting estimator (see Section 2.2.3 in [16]) which is sometimes referred to as time-continuous least squares estimator as well.

The crucial point of this work is the fact that, unlike the maximum likelihood estimator, our estimator requires the computation of integrals of the form (2) which can be calculated from the given data and which do not require further investigation determining the continuous part of the sample path.

In Sections 5 and 6 we prove strong consistency of our time-continuous least squares estimator for a time-inhomogeneous, mean-reverting Ornstein-Uhlenbeck process of the form (4) provided with a periodic drift. The case of a continuous driving process in this OrnsteinUhlenbeck model is studied by the authors in [7]. Note that mean reversion, periodicity and the occurrence of jumps are meaningful properties of, for example, energy commodity and particulary electricity data, cf. [10]. In the case of the ordinary Ornstein-Uhlenbeck process with jumps, [11] studies the large sample behavior of the time-continuous trajectory fitting estimator, various time-discrete estimation techniques are investigated in [4],[8], [12] and $[21]$.

\section{DesCRIPTION OF THE MODEL}

Suppose we are given a filtered probability space $\left(\Omega, \mathcal{F},\left(\mathcal{F}_{t}\right)_{t \geq 0}, P\right)$ where $\mathcal{F}_{0}$ contains all $P$-null sets of $\mathcal{F}$ and where $\mathcal{F}_{t}$ is right continuous, i.e. $\mathcal{F}_{t}=\cap_{u>t} \mathcal{F}_{u}$. Let $\left(L_{t}\right)_{t \geq 0}$ be a Lévy process, that is an $\mathcal{F}_{t}$-adapted process which is continuous in probability and which has independent and stationary increments. Throughout this work we want to consider its unique càdlàg (right-continuous with left limits) modification. The famous Lévy-Itô decomposition 
([1], Theorem 2.4.16 on p. 108) gives the path-wise representation

$$
L_{t}=b t+\sigma B_{t}+\int_{0}^{t} \int_{|x|<1} x \tilde{q}_{L}(d t, d x)+\int_{0}^{t} \int_{|x| \geq 1} x q_{L}(d t, d x)
$$

where $\left(B_{t}\right)_{t \geq 0}$ is a (standard) Brownian motion, $b \in \mathbb{R}, \sigma>0$ and where $q_{L}$ denotes the Poisson random measure associated with $\left(L_{t}\right)_{t \geq 0}$ while $\tilde{q}_{L}$ is the corresponding compensated measure. In detail, $q_{L}$ is a random measure on $\mathbb{R}_{+} \times(\mathbb{R} \backslash\{0\})$ defined by

$$
q_{L}(t, A)=\#\left\{0 \leq s \leq t: \Delta L_{s} \in A\right\}=\sum_{0 \leq s \leq t} \mathbf{1}_{A}\left(\Delta L_{s}\right)
$$

for all Borel sets $A \in \mathbb{R} \backslash\{0\}$. Thereby, we use the notation $\Delta L_{s}=L_{s}-L_{s-}, L_{s-}=$ $\lim _{\varepsilon \rightarrow 0} L_{s-\varepsilon}$ and $\mathbf{1}_{A}$ for the indicator function of the set $A$. Further, the compensated Poisson random measure is given by $\tilde{q}_{L}(d t, d x)=q_{L}(d t, d x)-d t \mu(d x)$ where $\mu$ is the Lévy measure associated with $\left(L_{t}\right)_{t \geq 0}$ satisfying $\int_{\mathbb{R} \backslash\{0\}}\left(x^{2} \wedge 1\right) \mu(d x)<\infty$. It holds that $b=E\left(L_{1}\right)-$ $\int_{|x| \geq 1} x \mu(d x)$.

The model of interest is the jump diffusion process $\left(X_{t}\right)_{t \geq 0}$ solving the stochastic differential equation

$$
d X_{t}=f\left(t, X_{t}\right) \theta d t+d L_{t}, \quad X_{0}=\xi
$$

where

$$
f(t, x)=\left(f_{1}(t, x), \ldots, f_{p}(t, x)\right), \quad p \in \mathbb{N}
$$

and where each $f_{i}(t, x)$ is a known, real-valued function on $[0, \infty) \times \mathbb{R}$. Further, let the random variable $\xi$ be independent of the Lévy process and $E\left(\xi^{2}\right)<\infty$. The drift parameter $\theta \in \mathbb{R}^{p}$ is unknown and has to be estimated. We require that the distribution of $\xi$ does not depend on $\theta$ otherwise the Radon-Nikodym derivative given in Proposition 4.2 would contain an additional factor, see [16] (p. 37) for details.

Note that equation (6) is a short form of the integral equation

$$
X_{t}-\xi=\int_{0}^{t} f\left(s, X_{s}\right) \theta d s+L_{t}
$$

We implicitly assume that the well-known Lipschitz and linear growth conditions on the drift function $f$ are satisfied (see [13], Theorem III.2.32 on p. 145) such that a unique càdlàg solution to (6) exists.

\section{Least SquARES ESTIMATOR}

In this section we introduce a least squares estimator for the drift parameter $\theta$. The derivation is based on a discretization of the stochastic differential equation (6) to which the ordinary least squares approach is applied. Taking the limit as the refinement improves yields a time-continuous estimator.

The stochastic differential equation (6) can be discretized on a time interval $[0, T]$ to the difference equation

$$
X_{(i+1) \Delta t}-X_{i \Delta t}=f\left(i \Delta t, X_{i \Delta t}\right) \theta \Delta t+\left(L_{(i+1) \Delta t}-L_{i \Delta t}\right), \quad i=0,1, \ldots, N,
$$

where $N=\lfloor T / \Delta t\rfloor-1$ and where $\Delta t>0$ denotes the constant time increment. Here $\lfloor x\rfloor$ denotes the integer part of $x$. The structure of (7) is similar to that of the classical linear model. Even though the conditions of the Gauss-Markov Theorem for linear models are not 
fulfilled we want to apply least squares estimation which is based on the minimization of the functional

$$
g: \theta \mapsto \sum_{i=0}^{N}\left(X_{(i+1) \Delta t}-X_{i \Delta t}-f\left(i \Delta t, X_{i \Delta t}\right) \theta \Delta t\right)^{2}
$$

Lemma 3.1. The solution vector $\tilde{\theta}_{T, \Delta t}$ to the minimization problem $g(\theta) \rightarrow$ min is given by

$$
\tilde{\theta}_{T, \Delta t}=Q_{T, \Delta t}^{-1} R_{T, \Delta t}
$$

where $Q_{T, \Delta t}=\left(\sum_{i=0}^{N} f_{j}\left(i \Delta t, X_{i \Delta t}\right) f_{k}\left(i \Delta t, X_{i \Delta t}\right) \Delta t\right)_{1 \leq j, k \leq p} \in \mathbb{R}^{p \times p}$ and

$R_{T, \Delta t}=\left(\sum_{i=0}^{N} f_{1}\left(i \Delta t, X_{i \Delta t}\right)\left(X_{(i+1) \Delta t}-X_{i \Delta t}\right), \ldots, \sum_{i=0}^{N} f_{p}\left(i \Delta t, X_{i \Delta t}\right)\left(X_{(i+1) \Delta t}-X_{i \Delta t}\right)\right)^{t} \in \mathbb{R}^{p}$.

Proof. By general theory of least squares estimation in linear models, the solution to the minimization problem $g(\theta) \rightarrow$ min is given by

$$
\theta_{\min }=\left(A^{t} A\right)^{-1} A^{t} D
$$

where

$$
A=\Delta t\left(\begin{array}{ccc}
f_{1}\left(0, X_{0}\right) & \cdots & f_{p}\left(0, X_{0}\right) \\
f_{1}\left(\Delta t, X_{\Delta t}\right) & \cdots & f_{p}\left(\Delta t, X_{\Delta t}\right) \\
\vdots & \ddots & \vdots \\
f_{1}\left(N \Delta t, X_{N \Delta t}\right) & \cdots & f_{p}\left(N \Delta t, X_{N \Delta t}\right)
\end{array}\right), \quad D=\left(\begin{array}{c}
X_{\Delta t}-X_{0} \\
X_{2 \Delta t}-X_{\Delta t} \\
\vdots \\
X_{(N+1) \Delta t}-X_{N \Delta t}
\end{array}\right)
$$

Hence, the products in equation (8) can be calculated to be

$$
A^{t} D=\Delta t R_{T, \Delta t}
$$

and

$$
A^{t} A=\Delta t Q_{T, \Delta t}
$$

Thus we get $\tilde{\theta}_{T, \Delta t}=\left(A^{t} A\right)^{-1} A^{t} D=Q_{T, \Delta t}^{-1} R_{T, \Delta t}$.

Now a continuous-time estimator can be derived from the least squares estimator by considering $\Delta t \rightarrow 0$. Note that any càdlàg function can be uniformly approximated on finite intervals by a sequence of step functions since it has countably many discontinuities on finite intervals. Hence, it is Riemann-integrable. This justifies the following convergence of the entries of $Q_{T, \Delta t}($ as $\Delta t \rightarrow 0)$ :

$$
\sum_{i=0}^{N} f_{l}\left(i \Delta t, X_{i \Delta t}\right) f_{m}\left(i \Delta t, X_{i \Delta t}\right) \Delta t \rightarrow \int_{0}^{T} f_{l}\left(t, X_{t}\right) f_{m}\left(t, X_{t}\right) d t
$$

since $f_{j}\left(t, X_{t}\right)$ has càdlàg paths (because $X_{t}$ has càdlàg paths) and the left-hand side of $(9)$ is a Riemann sum. Regarding the entries of $R_{T, \Delta t}$ it holds that

$$
\sum_{i=0}^{N} f_{j}\left(i \Delta t, X_{i \Delta t}\right) \cdot\left(X_{(i+1) \Delta t}-X_{i \Delta t}\right) \rightarrow \int_{0}^{T} f_{j}\left(t, X_{t-}\right) d X_{t}
$$

uniformly on compacts in probability since $X_{t}$ is a semi-martingale with càdlàg paths, see [18] (Theorem II.21 on p. 64).

We have thus proved the following proposition. 
Proposition 3.2. As $\Delta t \rightarrow 0$, the least squares estimator $\tilde{\theta}_{T, \Delta t}$ converges in probability to $\hat{\theta}_{T}=Q_{T}^{-1} R_{T}$ where $Q_{T}=\left(\int_{0}^{T} f_{j}\left(t, X_{t}\right) f_{k}\left(t, X_{t}\right) d t\right)_{1 \leq j, k \leq p} \in \mathbb{R}^{p \times p}$ and

$$
R_{T}=\left(\int_{0}^{T} f_{1}\left(t, X_{t-}\right) d X_{t}, \ldots, \int_{0}^{T} f_{p}\left(t, X_{t-}\right) d X_{t}\right)^{t} \in \mathbb{R}^{p} .
$$

We call the estimator $\hat{\theta}_{T}$ continuous-time least squares estimator.

Remark 1. We implicitly assumed that $Q_{T}$ is invertible. This condition holds for many reasonable models, like for jump diffusions of Ornstein-Uhlenbeck form. However, in the case of a singular matrix $Q_{T}$, one has to find solutions $\gamma \in \mathbb{R}^{p}$ to the normal equations

$$
Q_{T} \gamma=R_{T}
$$

and make further constraints in order to determine a proper estimator. Note that we have the same possible ambiguity of the solution vector in the case of maximum likelihood estimation which is presented in the next section.

\section{MAXimum LIKELIHOOd ESTIMATION}

We want to demonstrate the practical advantage of the continuous-time least squares estimator introduced in the previous section over the maximum likelihood estimator. In order to do so, we require the Lévy process which drives the jump diffusion given in (6) to fulfill the quite strong conditions (10) and (11), see below. These constraints are needed for technical reasons and the same shortcoming of the maximum likelihood estimator holds if (10) and (11) are not satisfied, see Remark 3.

Let $D[0, T]$ denote the space of càdlàg functions from $[0, T]$ to $\mathbb{R}$. Denote by $P_{X}$ and $P_{L}$ the measures induced by the process $\left(X_{t}\right)_{0 \leq t \leq T}$ solving equation (6) and by the Lévy process $\left(L_{t}\right)_{0 \leq t \leq T}$, respectively. That is

$$
P_{X}(B)=P\left(\omega: X^{T}(\omega) \in B\right), \quad P_{L}(B)=P\left(\omega: L^{T}(\omega) \in B\right)
$$

for all Borel sets $B \in D[0, T], X^{T}(\omega)=\left\{X_{t}(\omega), 0 \leq t \leq T\right\}$.

The following lemma is a direct application of Theorem 2.1 in [20] (p. 74).

Lemma 4.1. If $\int_{0}^{T}\left(f\left(t, X_{t}\right) \theta\right)^{2} d t<\infty P_{X^{-}}$and $P_{L^{-}}$almost surely for all $\theta$, then $P_{X}$ and $P_{L}$ are equivalent.

Let the Lévy process satisfy

$$
E\left(\exp \left(p L_{1}\right)\right)<\infty
$$

for all $p \in \mathbb{R}$. This condition guarantees the existence of moments of all orders of the Lévy process and the finiteness of $\int_{|x| \geq 1} x \mu(d x)$ allowing for the representation

$$
L_{t}=\sigma B_{t}+\int_{0}^{t} \int_{\mathbb{R}} x \tilde{q}_{L}(d t, d x)+t E\left(L_{1}\right) .
$$

We further assume that

$$
\int_{|x|<1}|x| \mu(d x)<\infty
$$


which implies finiteness of the total variation of almost all sample paths $t \mapsto\left(L_{t}-\sigma B_{t}\right)$ such that the sum of absolute 'small' jumps $\sum_{s \leq t}\left|\Delta L_{s}\right| \mathbf{1}_{\left\{\left|\Delta L_{s}\right|<1\right\}}\left(\Delta L_{s}\right)$ is convergent for almost every path.

Referring to Theorem 3.2 in [5] we have the following statement:

Proposition 4.2. Let $P_{X}$ be absolutely continuous with respect to $P_{L}$ and let

$$
E_{L}\left(\exp \left(\int_{0}^{T} f\left(t, \xi_{t-}\right) \theta d \xi_{t}^{c}-\frac{1}{2} \int_{0}^{T}\left(f\left(t, \xi_{t}\right) \theta\right)^{2} d t\right)\right)<\infty
$$

where $E_{L}$ denotes expectation with respect to $P_{L}$ and where $\left(\xi_{t}\right)_{0 \leq t \leq T}$ is an element in the sample space generated by the process solving (6) with a Lévy process satisfying (10) and (11). Further, the function $\xi_{t}^{c}=\xi_{t}-\sum_{s \leq t} \Delta \xi_{s}$ is the continuous part of the sample path $\xi_{t}$. Then the Radon-Nikodym derivative $d P_{X} / d P_{L}$ is given by

$$
\frac{d P_{X}}{d P_{L}}(\xi)=\exp \left(\int_{0}^{T} f\left(t, \xi_{t-}\right) \theta d \xi_{t}^{c}-\frac{1}{2} \int_{0}^{T}\left(f\left(t, \xi_{t}\right) \theta\right)^{2} d t\right)
$$

Remark 2. In our model specified in (6), the Radon-Nikodym derivative has a simpler form than the one in [5] because $X^{T}(\omega)$ and $L^{T}(\omega)$ exhibit the same jumps since

$$
\Delta X_{t}=\lim _{\varepsilon \rightarrow 0}\left(X_{t}-X_{t-\varepsilon}\right)=\lim _{\varepsilon \rightarrow 0} \int_{t-\varepsilon}^{t} d X_{t}=\lim _{\varepsilon \rightarrow 0} \int_{t-\varepsilon}^{t} f\left(t, X_{t}\right) \theta d t+\lim _{\varepsilon \rightarrow 0}\left(L_{t}-L_{t-\varepsilon}\right)=\Delta L_{t} .
$$

Hence, it holds for the point process $q_{X}$ associated with $\left(X_{t}\right)_{t \geq 0}$ that

$$
q_{X}(t, A)=q_{L}(t, A)
$$

for all Borel sets $A \in \mathbb{R} \backslash\{0\}$ and all $t$. Consequently, the change of measure from $P_{L}$ to $P_{X}$ does not change the 'weights' of the discontinuities. So, in our framework, the density of $P_{X}$ with respect to $P_{L}$ does not include any term that accounts for the jumps.

Remark 3. The assumption (11) simplifies the representation of the continuous part of the sample path and is not required in [5]. Note that a more general statement without the need of (10) and (11) can be found in [20] (Theorem 2.1) whereby the corresponding integrator is again the continuous part of the sample path.

Suppose we observe a sample path $X^{T}=\left\{X_{t}, 0 \leq t \leq T\right\}$ of the process with stochastic differential given in (6). The maximum likelihood estimator $\check{\theta}_{T}$ is defined by

$$
\check{\theta}_{T}:=\arg \max _{\theta} \frac{d P_{X}}{d P_{L}}\left(X^{T}\right)
$$

where the Radon-Nikodym density $d P_{X} / d P_{L}$ obviously depends on the parameter $\theta$, see the previous proposition. The partial derivatives of the logarithm of this functional are of the form

$$
\frac{\partial}{\partial \theta_{i}} \ln \left(\frac{d P_{X}}{d P_{L}}\left(X^{T}\right)\right)=\int_{0}^{T} \frac{\partial}{\partial \theta_{i}} f\left(t, X_{t-}\right) \theta d X_{t}^{c}-\int_{0}^{T} f\left(t, X_{t}\right) \theta \frac{\partial}{\partial \theta_{i}} f\left(t, X_{t}\right) \theta d t
$$

$i=1, \ldots, p$, and the single derivatives of the linear drift function are given by $\frac{\partial}{\partial \theta_{i}} f\left(t, X_{t}\right) \theta=$ $f_{i}\left(t, X_{t}\right)$. Setting the derivatives in (12) equal zero results in a system of equations

$$
\int_{0}^{T} f_{i}\left(t, X_{t-}\right) d X_{t}^{c}-\int_{0}^{T} f\left(t, X_{t}\right) \check{\theta}_{T} f_{i}\left(t, X_{t}\right) d t=0, \quad i=1, \ldots, p,
$$


which can be written as

$$
\begin{aligned}
& \text { where } Q_{T}=\left(\int_{0}^{T} f_{j}\left(t, X_{t}\right) f_{k}\left(t, X_{t}\right) d t\right)_{1 \leq j, k \leq p} \in Q_{T} \check{\theta}_{T}=0 \\
& \qquad \tilde{R}_{T}=\left(\int_{0}^{T \times p}\right. \text { and } \\
& \left.f_{1}\left(t, X_{t-}\right) d X_{t}^{c}, \ldots, \int_{0}^{T} f_{p}\left(t, X_{t-}\right) d X_{t}^{c}\right)^{t} \in \mathbb{R}^{p} .
\end{aligned}
$$

Proposition 4.3. The maximum likelihood estimator $\check{\theta}_{T}$ is given by

$$
\check{\theta}_{T}=Q_{T}^{-1} \tilde{R}_{T}
$$

where $Q_{T}$ and $\tilde{R}_{T}$ are defined above.

Note that the expression for the maximum likelihood estimator $\check{\theta}_{T}$ is similar to the least squares estimator $\hat{\theta}_{T}$ given in Proposition 3.2. The discrepancy lies in the vectors $\tilde{R}_{T}$ and $R_{T}$. The entries of the latter are of the form $\int_{0}^{T} f_{i}\left(t, X_{t-}\right) d X_{t}$ and can be calculated in practice without any difficulty. If time-discrete observations are available these integrals can be

approximated by sums. In contrast to that, the integrals $\int_{0}^{T} f_{i}\left(t, X_{t-}\right) d X_{t}^{c}$ in $\tilde{R}_{T}$ cannot be computed without further investigation due to the integrator which is the continuous part of the sample path. In practice, a discontinuous path is observed such that the continuous part of this path has to be determined by means of further techniques detaching discontinuities. This is a challenging issue unless the paths of the Lévy process have a finite number of jumps along the time interval $[0, T]$. In the case of time-discrete observations the always arising problem is to distinguish the jumps from the continuous points since the entire time-discrete sample looks discontinuous.

Remark 4. In the case of an ordinary diffusion process without jumps, that is the process solving

$$
d X_{t}=f\left(t, X_{t}\right) \theta d t+d B_{t}, \quad X_{0}=\xi
$$

where $\left(B_{t}\right)_{t \geq 0}$ is a Brownian motion, the Radon-Nikodym derivative in Proposition 4.2 takes the form

$$
\frac{d P_{X}}{d P_{B}}\left(X^{T}\right)=\exp \left(\int_{0}^{T} f\left(t, X_{t}\right) \theta d X_{t}-\frac{1}{2} \int_{0}^{T}\left(f\left(t, X_{t}\right) \theta\right)^{2} d t\right)
$$

for data $X^{T}$. This expression is in accordance with the famous Girsanov Theorem, see [17] (Theorem 7.6, p. 246). The first integral is computed with respect to the entire path since there do not occur any discontinuities in this model. Note that the derivation of the maximum likelihood estimator goes in line with the considerations given above, i.e. differentiating and solving the resulting system of equations, such that $\check{\theta}_{T}=Q_{T}^{-1} R_{T}=\hat{\theta}_{T}$. That means, in this continuous diffusion model, the maximum likelihood method provides the same estimator as our least squares methodology presented in the previous section.

\section{Consistency of Least squares estimator}

In order to substantiate the convenience of the least squares estimator introduced in Section 3 we show (strong) consistency of this estimator for a concrete jump diffusion model. In the quite general setup with regard to the drift function $f$ in (6) consistency requires general conditions on the convergence of the matrix $Q_{T}$ in Proposition 3.2 which are not helpful for the application in concrete models. 
Let us consider the time-inhomogeneous, mean-reverting Ornstein-Uhlenbeck process with jumps which we define as the solution to

$$
d X_{t}=\Phi\left(t, X_{t}\right) \theta d t+d L_{t}, \quad X_{0}=\xi
$$

where

$$
\Phi(t, x)=\left(\varphi_{1}(t), \ldots, \varphi_{p}(t),-x\right), \quad p \in \mathbb{N},
$$

with known, real-valued functions $\varphi_{1}, \ldots, \varphi_{p}$ and $E\left(\xi^{2}\right)<\infty, \xi$ being independent of the Lévy process. We denote the parameter vector by $\theta=\left(\theta_{1}, \ldots \theta_{p}, \alpha\right)^{t} \in \mathbb{R}^{p} \times \mathbb{R}_{+}$. We assume that the drift function $\Phi$ is periodic in $t$, i.e.

$$
\Phi(t+\nu, x)=\Phi(t, x) \quad \text { for all } x
$$

where $\nu$ is a known period. Seasonality in the drift is a quite frequent phenomenon in applications, e.g. in commodity prices or temperature modeling. This assumed periodicity leads to the requirement $\varphi_{j}(t+\nu)=\varphi_{j}(t)$. Due to Gram-Schmidt orthogonalization, we may assume without loss of generality that $\varphi_{1}(t), \ldots, \varphi_{p}(t)$ form an orthonormal system in $L_{2}\left([0, \nu], \frac{1}{\nu} d \lambda\right)$, that means that

$$
\int_{0}^{\nu} \varphi_{j}(t) \varphi_{k}(t) d t= \begin{cases}\nu, & j=k \\ 0, & j \neq k\end{cases}
$$

Henceforth, we will assume that we observe an integral multiple of the period length, i.e. that

$$
T=N \nu
$$

for some integer $N$. Moreover, we will assume without loss of generality that $\nu=1$.

The driving process $\left(L_{t}\right)_{t \geq 0}$ is the right-continuous modification of a Lévy process of the form as described in Section 2, that is, as usual, a stochastic process that is continuous in probability with stationary and independent increments. For this model, we additionally require

$$
E\left(L_{t}^{2}\right)<\infty
$$

for all $t$ which is equivalent to the requirement $\int_{|x|>1} x^{2} \mu(d x)<\infty$ where $\mu$ denotes the Lévy measure. Further, we consider a centered process, that is

$$
E\left(L_{1}\right)=0
$$

implying that $\left(L_{t}\right)_{t \geq 0}$ is a martingale.

Note that under the assumptions (14) and (15) the matrix $Q_{T}$ in Proposition 3.2 simplifies to

$$
Q_{T}=\left(\begin{array}{cc}
T I_{p} & -a_{T} \\
-a_{T}^{t} & b_{T}
\end{array}\right)
$$

where $a_{T}=\left(\int_{0}^{T} \varphi_{1}(t) X_{t} d t, \ldots, \int_{0}^{T} \varphi_{p}(t) X_{t} d t\right)^{t}, b_{T}=\int_{0}^{T} X_{t}^{2} d t$ and where $I_{p}$ denotes the $(p \times p)$-identity matrix.

The main result of this section can be formulated now. Its proof is postponed to Section 6 . 
Theorem 1. Let $\left\{X_{t}, 0 \leq t \leq T\right\}$ be observations of the periodic Ornstein-Uhlenbeck process introduced above satisfying (14), (15), (16) and (17). Then the least squares estimator given in Proposition 3.2 is consistent, i.e.

$$
\hat{\theta}_{T} \rightarrow \theta, \text { almost surely, }
$$

as $T \rightarrow \infty$.

Remark 5. In the particular case where the driving process is Brownian motion, asymptotic normality of the least squares estimator is proved in [7] (Theorem 2). In that continuous model, least squares and maximum likelihood give the same estimator, see Remark 4 .

\section{Proof of CONSISTENCY}

The following representation of the least squares estimator is essential for the proof:

Proposition 6.1. The least squares estimator $\hat{\theta}_{T}$ can be written as

$$
\hat{\theta}_{T}=\theta+Q_{T}^{-1} S_{T}
$$

where

$$
S_{T}=\left(\begin{array}{c}
\int_{0}^{T} \varphi_{1}(t) d L_{t} \\
\vdots \\
\int_{0}^{T} \varphi_{p}(t) d L_{t} \\
-\int_{0}^{T} X_{t-} d L_{t}
\end{array}\right)
$$

and where $Q_{T}$ is given in (18).

Proof. By definition, we have $\hat{\theta}_{T}=Q_{T}^{-1} R_{T}$ where $Q_{T}$ is given in (18) and

$$
R_{T}=\left(\begin{array}{c}
\int_{0}^{T} \varphi_{1}(t) d X_{t} \\
\vdots \\
\int_{0}^{T} \varphi_{p}(t) d X_{t} \\
-\int_{0}^{T} X_{t-} d X_{t}
\end{array}\right)
$$

in the model considered here, see Proposition 3.2. Due to the stochastic differential equation (13) which is generating the data and which can be written as

$$
d X_{t}=\left(\sum_{j=1}^{p} \theta_{j} \varphi_{j}(t)-\alpha X_{t}\right) d t+d L_{t}
$$

the stochastic integrals in $R_{T}$ are seen to be

$$
\begin{aligned}
\int_{0}^{T} \varphi_{i}(t) d X_{t} & =\sum_{j=1}^{p} \theta_{j} \int_{0}^{T} \varphi_{i}(t) \varphi_{j}(t) d t-\alpha \int_{0}^{T} \varphi_{i}(t) X_{t} d t+\int_{0}^{T} \varphi_{i}(t) d L_{t}, \quad i=1, \ldots, p \\
\int_{0}^{T} X_{t-} d X_{t} & =\sum_{j=1}^{p} \theta_{j} \int_{0}^{T} \varphi_{j}(t) X_{t} d t-\alpha \int_{0}^{T} X_{t}^{2} d t+\int_{0}^{T} X_{t-} d L_{t} .
\end{aligned}
$$

Observe that the set $\left\{t \in[0, T]: X_{t} \neq X_{t-}\right\}$ is countable and has thus zero-mass with respect to $d t$. Hence, we can conclude from these representations combined with (14) and (15) that

$$
R_{T}=\left(\begin{array}{cc}
T I_{p} & -a_{T} \\
-a_{T}^{t} & b_{T}
\end{array}\right) \theta+S_{T}
$$


and it follows that $Q_{T}^{-1} R_{T}=\theta+Q_{T}^{-1} S_{T}$.

Due to representation (19) the aim in the sequel is to show that

$$
Q_{T}^{-1} S_{T}=\left(T Q_{T}^{-1}\right)\left(\frac{1}{T} S_{T}\right)
$$

converges to zero almost surely as $T \rightarrow \infty$. Therefor, we will prove that $T Q_{T}^{-1}$ converges to a finite limit and that $\frac{1}{T} S_{T}$ tends to zero, almost surely respectively. Both of these results require some auxiliary results.

Lemma 6.2. The solution to the stochastic differential equation (13) is explicitly given by

$$
X_{t}=e^{-\alpha t} X_{0}+h(t)+Z_{t}
$$

where

$$
h(t)=e^{-\alpha t} \sum_{i=1}^{p} \theta_{i} \int_{0}^{t} e^{\alpha s} \varphi_{i}(s) d s
$$

and

$$
Z_{t}=e^{-\alpha t} \int_{0}^{t} e^{\alpha s} d L_{s}
$$

Proof. The Itô lemma, see [18] (Theorem II.32, p. 71) or [14] (Theorem 7.6.1 and 7.6.4, p. 111 and 113), states that it holds for a semimartingale $\left(Y_{t}\right)_{t \geq 0}$ and a $C^{1,2}$ function $F(t, x)$ that

$$
\begin{aligned}
F\left(t, Y_{t}\right)= & F\left(0, Y_{0}\right)+\int_{0}^{t} \frac{\partial F}{\partial t}\left(s, Y_{s}\right) d s+\int_{0}^{t} \frac{\partial F}{\partial x}\left(s, Y_{s-}\right) d Y_{s}+\frac{1}{2} \int_{0}^{t} \frac{\partial^{2} F}{\partial x^{2}}\left(s, Y_{s-}\right) d[Y]_{s}^{c} \\
& +\sum_{0<s \leq t}\left(F\left(s, Y_{s}\right)-F\left(s, Y_{s-}\right)-\frac{\partial F}{\partial x}\left(s, Y_{s-}\right) \Delta Y_{s}\right)
\end{aligned}
$$

where $[Y]_{s}^{c}$ is the continuous part of the quadratic variation process $[Y]_{s}$ and $\Delta Y_{s}=Y_{s}-Y_{s-}$.

Now for $F(t, x)=e^{\alpha t} x$ and the $\operatorname{SDE}(13)$ which can be written as

$$
d X_{t}=\left(\sum_{j=1}^{p} \theta_{j} \varphi_{j}(t)-\alpha X_{t}\right) d t+d L_{t}
$$

we obtain

$$
\begin{aligned}
F\left(t, X_{t}\right) & =X_{0}+\int_{0}^{t} \alpha e^{\alpha s} X_{s} d s+\int_{0}^{t} e^{\alpha s} d X_{s}+\sum_{0<s \leq t}\left(e^{\alpha s} X_{s}-e^{\alpha s} X_{s-}-e^{\alpha s} \Delta X_{s}\right) \\
& =X_{0}+\sum_{j=1}^{p} \theta_{j} \int_{0}^{t} e^{\alpha s} \varphi_{j}(s) d s+\int_{0}^{t} e^{\alpha s} d L_{s}
\end{aligned}
$$

by making use of the equality $X_{s}-X_{s-}=\Delta X_{s}$. Multiplying by $e^{-\alpha t}$ finishes the proof.

Due to the time-dependence of $h$ and $Z$ in $(21)$ the process $\left(X_{t}\right)_{t \geq 0}$ is not stationary in the ordinary sense such that the ergodic theorem is not directly applicable. We will introduce a solution to the stochastic differential equation (13) with time index $t \in \mathbb{R}$ instead of $t \geq 0$. By interpreting this process as a sequence of path-valued random variables we prove stationarity and ergodicity of this sequence. 
Define the process

$$
\tilde{X}_{t}=\tilde{h}(t)+\tilde{Z}_{t}
$$

where $\tilde{h}:[0, \infty) \rightarrow \mathbb{R}$ is defined by

$$
\tilde{h}(t)=e^{-\alpha t} \sum_{j=1}^{p} \theta_{j} \int_{-\infty}^{t} e^{\alpha s} \varphi_{j}(s) d s
$$

and

$$
\tilde{Z}_{t}=e^{-\alpha t} \int_{-\infty}^{t} e^{\alpha s} d \tilde{L}_{s}
$$

whereby

$$
\tilde{L}_{s}:=L_{s} \mathbf{1}_{\{s \geq 0\}}(s)+\bar{L}_{s} \mathbf{1}_{\{s<0\}}(s)
$$

by taking $\bar{L}_{s}$, when $s<0$, to be an independent copy of $-L_{-(s-)}$ (see p. 214 in [1]). Constructed in this way, the process $\left(\tilde{L}_{t}\right)_{t \in \mathbb{R}}$ is a continuation of $\left(L_{t}\right)_{t \geq 0}$ to $\mathbb{R}$ such that $\left(\tilde{L}_{t}\right)_{t \in \mathbb{R}}$ is also a Lévy process with càdlàg paths.

Let $D[0,1]$ be the space of càdlàg functions on $[0,1]$.

Lemma 6.3. The sequence $\left(W_{k}\right)_{k \in \mathbb{N}}$ of $D[0,1]$-valued random variables defined by

$$
W_{k}(s):=\tilde{X}_{k-1+s}, \quad 0 \leq s \leq 1
$$

is stationary and ergodic.

Proof. Let $\tilde{h}_{0}$ be the restriction of the function $\tilde{h}$ to $[0,1]$. Since $\tilde{h}$ is periodic, we have the decomposition

$$
\begin{aligned}
W_{k}(t) & =\tilde{h}(k-1+t)+e^{-\alpha(k-1+t)} \int_{-\infty}^{k-1+t} e^{\alpha s} d \tilde{L}_{s} \\
& =\tilde{h}_{0}(t)+e^{-\alpha(k-1+t)} \int_{k-1}^{k-1+t} e^{\alpha s} d \tilde{L}_{s}+\sum_{l=-\infty}^{k-1} e^{-\alpha(k-1+t)} \int_{l-1}^{l} e^{\alpha s} d \tilde{L}_{s} .
\end{aligned}
$$

The time shifted Lévy martingale $\tilde{L}_{s}^{(l)}:=\tilde{L}_{s+l}$ yields

$$
\begin{aligned}
W_{k}(t) & =\tilde{h}_{0}(t)+e^{-\alpha t} \int_{0}^{t} e^{\alpha s} d \tilde{L}_{s}^{(k-1)}+\sum_{l=-\infty}^{k-1} e^{-\alpha(k-l+t)} \int_{0}^{1} e^{\alpha s} d \tilde{L}_{s}^{(l-1)} \\
& =\tilde{h}_{0}(t)+e^{-\alpha t} \int_{0}^{t} e^{\alpha s} d \tilde{L}_{s}^{(k-1)}+\sum_{j=-\infty}^{0} e^{-\alpha(1+t-j)} \int_{0}^{1} e^{\alpha s} d \tilde{L}_{s}^{(j+k-2)} .
\end{aligned}
$$

Consequently, we can write

$$
W_{k}(\cdot)=\tilde{h}_{0}(\cdot)+F_{0}\left(Y_{k-1}\right)+\sum_{l=-\infty}^{0} e^{\alpha(j-1)} F\left(Y_{j+k-2}\right)
$$

by using the almost surely defined functionals

$$
F_{0}: D[0,1] \rightarrow D[0,1] ; \omega \mapsto\left(t \mapsto e^{-\alpha t} \int_{0}^{t} e^{\alpha s} d \omega(s)\right),
$$




$$
F: D[0,1] \rightarrow D[0,1] ; \omega \mapsto\left(t \mapsto e^{-\alpha t} \int_{0}^{1} e^{\alpha s} d \omega(s)\right)
$$

and the $D[0,1]$-valued random variables

$$
Y_{l}: s \mapsto\left(\tilde{L}_{s}^{(l)}-\tilde{L}_{0}^{(l)}\right), 0 \leq s<1 .
$$

The sequence $\left(Y_{l}\right)_{l \in \mathbb{Z}}$ consists of independent and identically distributed random variables. This implies that $\left(W_{k}\right)_{k \in \mathbb{N}}$ is stationary and ergodic since each element of this sequence can be represented as a measurable function $G:(D[0,1])^{\mathbb{N}} \rightarrow D[0,1]$ of elements of the iid sequence $\left(Y_{l}\right)_{l \in \mathbb{Z}}$, i.e.

$$
W_{k}=G\left(Y_{k}, Y_{k-1}, \ldots\right)
$$

The conditions (16) and(17) are incorporated into the next lemma. Denote by $\left(\langle L\rangle_{t}\right)_{t \geq 0}$ the uniquely determined previsible process with non-decreasing sample paths such that

$$
\left(L_{t}^{2}-\langle L\rangle_{t}\right)_{t \geq 0}
$$

is a martingale. Usually $\left(\langle L\rangle_{t}\right)_{t \geq 0}$ is referred to as compensator or bracket process associated with $(L)_{t \geq 0}$. Its existence is guaranteed by the Doob-Meyer decomposition of $L_{t}^{2}$.

Lemma 6.4. Let $\left(L_{t}\right)_{t \geq 0}$ be a Lévy process satisfying (16) and (17). Then the bracket process $\left(\langle L\rangle_{t}\right)_{t \geq 0}$ in (25) is of the form

$$
\langle L\rangle_{t}=c t, \quad t \geq 0
$$

where $c$ is a finite, non-negative constant.

Proof. By the Doob-Meyer decomposition the process $\left(L_{t}^{2}-\langle L\rangle_{t}\right)_{t \geq 0}$ is a martingale. It can be proved that $\left(L_{t}^{2}-[L]_{t}\right)_{t \geq 0}$ is also a martingale, where $\left([L]_{t}\right)_{t \geq 0}$ denotes the quadratic variation process. Hence, $\left([\bar{L}]_{t}-\langle L\rangle_{t}\right)_{t \geq 0}$ is a martingale. Further, the process $\left([L]_{t}\right)_{t \geq 0}$ is a Lévy process implying that $\left([L]_{t}-E\left([L]_{t}\right)\right)_{t \geq 0}$ is a martingale. According to [6] (Example III.8.5, p. 266) the quadratic variation process has the form

$$
[L]_{t}=\sigma^{2} t+\int_{0}^{t} \int_{\mathbb{R} \backslash\{0\}} x^{2} q_{L}(d t, d x)
$$

where $q_{L}$ denotes the jump measure associated with $\left(L_{t}\right)_{t \geq 0}$. Since by (16) and the fundamental properties of the Lévy measure, see Section 2, it holds that $\int_{\mathbb{R} \backslash\{0\}} x^{2} \mu(d x)<\infty$ and $E\left(q_{L}(d t, d x)\right)=d t \mu(d x)$, it follows that $E\left([L]_{t}\right)=c t$.

Lemma 6.5. Ast $\rightarrow \infty$, one has

$$
\left|\tilde{X}_{t}-X_{t}\right| \rightarrow 0, \quad \text { almost surely } .
$$

Proof. We have

$$
\begin{aligned}
\left|\tilde{X}_{t}-X_{t}\right| & \leq e^{-\alpha t}\left|X_{0}\right|+|\tilde{h}(t)-h(t)|+\left|\tilde{Z}_{t}-Z_{t}\right| \\
& \leq e^{-\alpha t}\left|X_{0}\right|+e^{-\alpha t} \sum_{i=1}^{p} \theta_{i} \int_{-\infty}^{0} e^{\alpha s}\left|\varphi_{i}(s)\right| d s+\left|e^{-\alpha t} \int_{-\infty}^{0} e^{\alpha s} d \tilde{L}_{s}\right|
\end{aligned}
$$


Obviously, the first two terms on the right-hand side converge toward zero as $t \rightarrow \infty$. Further, since $E\left(L_{t}^{2}\right)<\infty$ by assumption, it holds by the Itô isometry for stochastic integrals with martingales as integrators that

$$
E\left(\left(\int_{-\infty}^{0} e^{\alpha s} d \tilde{L}_{s}\right)^{2}\right)=E\left(\int_{-\infty}^{0} e^{2 \alpha s} d\langle\tilde{L}\rangle_{s}\right)
$$

where $\langle\tilde{L}\rangle_{s}$ denotes the compensator of the Lévy martingale $\tilde{L}_{s}$ obtained from the DoobMeyer decomposition such that $\left(\tilde{L}_{s}^{2}-\langle\tilde{L}\rangle_{s}\right)_{s}$ is a martingale. By Lemma 6.4 it is $\langle\tilde{L}\rangle_{s}=c s$, where $c$ is a finite, non-negative constant, so that

$$
E\left(\int_{-\infty}^{0} e^{2 \alpha s} d\langle\tilde{L}\rangle_{s}\right)=\int_{-\infty}^{0} e^{2 \alpha s} c d s=\frac{c}{2 \alpha}<\infty .
$$

Hence we have shown that $E\left(\int_{-\infty}^{0} e^{\alpha s} d \tilde{L}_{s}\right)^{2}<\infty$ which implies that $\left|\int_{-\infty}^{0} e^{\alpha s} d \tilde{L}_{s}\right|<\infty$ almost surely. It follows that

$$
e^{-\alpha t}\left|\int_{-\infty}^{0} e^{\alpha s} d \tilde{L}_{s}\right| \rightarrow 0
$$

as $t \rightarrow \infty$.

Let us now turn to the matrix $Q_{T}$. Due to its simplified form in this model, see representation (18), its inverse can be explicitly computed.

Lemma 6.6. The inverse of the matrix $Q_{T}$ given in (18) can be computed to be

$$
Q_{T}^{-1}=\frac{1}{T}\left(\begin{array}{cc}
I_{p}+\gamma_{T} \Lambda_{T} \Lambda_{T}^{t} & -\gamma_{T} \Lambda_{T} \\
-\gamma_{T} \Lambda_{T}^{t} & \gamma_{T}
\end{array}\right)
$$

where $\Lambda_{T}=\left(\Lambda_{T, 1}, \ldots, \Lambda_{T, p}\right)^{t}=\frac{1}{T} a_{T}$, see (18), and

$$
\gamma_{T}=\left(\frac{1}{T} \int_{0}^{T} X_{t}^{2} d t-\sum_{i=1}^{p} \Lambda_{T, i}^{2}\right)^{-1} .
$$

Proof. We make use of a formula for the inverse of a partitioned matrix, also known as Frobenius matrix inversion formula. It can be found in [9] (p. 73) or one can verify it directly. It holds for $a \in \mathbb{R}^{p}, b \in \mathbb{R}$ that

$$
\left(\begin{array}{ll}
I_{p} & a \\
a^{t} & b
\end{array}\right)^{-1}=\left(\begin{array}{cc}
I_{p}+\frac{1}{b-\|a\|^{2}} a a^{t} & -\frac{1}{b-\|a\|^{2}} a \\
-\frac{1}{b-\|a\|^{2}} a^{t} & \frac{1}{b-\|a\|^{2}}
\end{array}\right)
$$

where $\|\cdot\|$ denotes the usual Euclidean norm on $\mathbb{R}^{p}$. With the notation introduced above, we can write $Q_{T}$ as

$$
Q_{T}=T\left(\begin{array}{cc}
I_{p} & -\Lambda_{T} \\
-\Lambda_{T}^{t} & \frac{1}{T} \int_{0}^{T} X_{t}^{2} d t
\end{array}\right)
$$

and thus apply the above formula for the calculation of $Q_{T}^{-1}$. 
Remark 6. Note that the Frobenius matrix inversion formula holds if and only if the entries of the matrix on the right hand side of (27) are well-defined. We will see in the proof of Proposition 6.7 that the limit of $\frac{1}{T} Q_{T}^{-1}$ is well defined since we show that the limit of $\gamma_{T}$ denoted by $\gamma$ is greater than zero. Consequently, $\frac{1}{T} Q_{T}^{-1}$ exists almost surely if $T$ is sufficiently large.

Proposition 6.7. As $T \rightarrow \infty$, we have

$$
T Q_{T}^{-1} \rightarrow C, \quad \text { almost surely, }
$$

where $C$ is the $(p+1) \times(p+1)$ matrix

$$
C=\left(\begin{array}{cc}
I_{p}+\gamma \Lambda \Lambda^{t} & -\gamma \Lambda \\
-\gamma \Lambda^{t} & \gamma
\end{array}\right)
$$

whereby $\Lambda=\left(\Lambda_{1}, \ldots, \Lambda_{p}\right)^{t}$ and

$$
\begin{aligned}
\Lambda_{i} & =\int_{0}^{1} \varphi_{i}(t) \tilde{h}(t) d t, \quad i=1, \ldots, p \\
\gamma & =\left(\int_{0}^{1}(\tilde{h}(t))^{2} d t+E\left(\tilde{Z}_{0}^{2}\right)-\sum_{i=1}^{p} \Lambda_{i}^{2}\right)^{-1} .
\end{aligned}
$$

The function $\tilde{h}$ and the random variable $\tilde{Z}_{t}$ are specified in (23) and (24).

Proof. Consider the entries of the vector $\Lambda_{T}$ first, i.e. $\frac{1}{T} \int_{0}^{T} X_{t} \varphi_{j}(t) d t$. From Lemma 6.5 we may conclude that

$$
\frac{1}{T} \int_{0}^{T} \tilde{X}_{t} \varphi_{j}(t) d t-\frac{1}{T} \int_{0}^{T} X_{t} \varphi_{j}(t) d t \rightarrow 0
$$

almost surely. Since $\left(\tilde{X}_{k-1+s}\right)_{k \in \mathbb{N}}$ is stationary and ergodic by Lemma 6.3 , the ergodic theorem justifies

$$
\frac{1}{T} \int_{0}^{T} \tilde{X}_{t} \varphi_{j}(t) d t=\frac{1}{T} \sum_{k=1}^{T} \int_{k-1}^{k} \tilde{X}_{t} \varphi_{j}(t) d t \rightarrow E\left(\int_{0}^{1} \tilde{X}_{t} \varphi_{j}(t) d t\right)=\int_{0}^{1} \tilde{h}(t) \varphi_{j}(t) d t
$$

almost surely. Thus we have established convergence of $\Lambda_{T, j}, 1 \leq j \leq p$. For the asymptotic behavior of $\gamma_{T}$, it suffices to investigate $\frac{1}{T} \int_{0}^{T} X_{t}^{2} d t$.

It holds by (26) that

$$
\left|\frac{1}{T} \int_{0}^{T}\left(Z_{t}-\tilde{Z}_{t}\right) d t\right| \leq \frac{1}{T} \int_{0}^{T}\left|Z_{t}-\tilde{Z}_{t}\right| d t=\frac{1}{T} \int_{0}^{T} e^{-\alpha t}\left|\int_{-\infty}^{0} e^{\alpha s} d \tilde{L}_{s}\right| d t \rightarrow 0,
$$

almost surely, as $T \rightarrow \infty$. The ergodic theorem gives

$$
\frac{1}{T} \int_{0}^{T} \tilde{Z}_{t} d t \rightarrow E\left(\tilde{Z}_{0}\right)=0
$$

compare the proof of Lemma 6.3 and we may conclude that

$$
\limsup _{T \rightarrow \infty} \frac{1}{T} \int_{0}^{T} Z_{t} d t<\infty .
$$


Observe that $h(t)$ is bounded and $X_{0}<\infty$ almost surely. These facts combined with (28) and representation (21) justify

$$
\limsup _{T \rightarrow \infty} \frac{1}{T} \int_{0}^{T} X_{t} d t=\limsup _{T \rightarrow \infty} \frac{1}{T} \int_{0}^{T}\left(e^{-\alpha t} X_{0}+h(t)+Z_{t}\right) d t<\infty
$$

almost surely. It follows from (29) and Lemma 6.5 that

$$
\frac{1}{T} \int_{0}^{T} \tilde{X}_{t}^{2} d t-\frac{1}{T} \int_{0}^{T} X_{t}^{2} d t=\frac{1}{T} \int_{0}^{T}\left(\tilde{X}_{t}+X_{t}\right)\left(\tilde{X}_{t}-X_{t}\right) d t \rightarrow 0 .
$$

Consequently, again by the ergodic theorem, we get

$$
\begin{aligned}
\frac{1}{T} \int_{0}^{T} \tilde{X}_{t}^{2} d t & =\frac{1}{T} \sum_{k=1}^{T} \int_{k-1}^{k} \tilde{X}_{t}^{2} d t \\
& \rightarrow E\left(\int_{0}^{1} \tilde{X}_{t}^{2} d t\right) \\
& =E\left(\int_{0}^{1}\left(\tilde{h}(t)+\tilde{Z}_{t}\right)^{2} d t\right) \\
& =E\left(\int_{0}^{1}(\tilde{h}(t))^{2} d t+2 \int_{0}^{1} \tilde{h}(t) \tilde{Z}_{t} d t+\int_{0}^{1} \tilde{Z}_{t}^{2} d t\right) \\
& =\int_{0}^{1}(\tilde{h}(t))^{2} d t+E\left(\tilde{Z}_{0}^{2}\right) .
\end{aligned}
$$

By Bessel's inequality, we have

$$
\sum_{i=1}^{p} \Lambda_{i}^{2} \leq \int_{0}^{1}(\tilde{h}(t))^{2} d t
$$

and thus $\left(\int_{0}^{1}(\tilde{h}(t))^{2} d t+E\left(\tilde{Z}_{0}^{2}\right)-\sum_{i=1}^{p} \Lambda_{i}^{2}\right) \geq E\left(\tilde{Z}_{0}^{2}\right)>0$.

Lemma 6.8. The term $\frac{1}{\sqrt{T}} S_{T}$ is bounded in $L^{2}$.

Proof. Note that $\frac{1}{\sqrt{T}} \int_{0}^{T} \varphi_{i}(t) d L_{t}$ is $L^{2}$-bounded since

$$
E\left(\frac{1}{\sqrt{T}} \int_{0}^{T} \varphi_{i}(t) d L_{t}\right)^{2}=E\left(\frac{1}{T} \int_{0}^{T} \varphi_{i}(t)^{2} d\langle L\rangle_{t}\right)=\frac{c}{T} \int_{0}^{T} \varphi_{i}(t)^{2} d t<\infty
$$

by making use of the Itô isometry and Lemma 6.4. For the last entry of $\frac{1}{\sqrt{T}} S_{T}$ we have to prove the boundedness of

$$
\begin{aligned}
& E\left(\frac{1}{\sqrt{T}} \int_{0}^{T} X_{t} d L_{t}\right)^{2}=\frac{1}{T} E\left(\int_{0}^{T} X_{t}^{2} d\langle L\rangle_{t}\right) \\
& =\frac{1}{T} E\left(\int_{0}^{T}\left(2 e^{-\alpha t} X_{0} h(t)+2 e^{-\alpha t} X_{0} Z_{t}+e^{-2 \alpha t} X_{0}^{2}+2 h(t) Z_{t}+h(t)^{2}+Z_{t}^{2}\right) d\langle L\rangle_{t}\right) .
\end{aligned}
$$

Again, $\langle L\rangle_{t}=$ ct. Since $Z_{t}$ is a zero-mean random variable the expectation of the second and fourth term is zero. Moreover, $E\left(Z_{t}^{2}\right)=\frac{c}{2 \alpha}\left(1-e^{-2 \alpha t}\right)<\infty$ such that

$$
\sup _{T \geq 0} \frac{1}{T} E\left(\int_{0}^{T} Z_{t}^{2} d t\right)<\infty \text {. }
$$


Further, the function $h$ is bounded and $E\left(X_{0}^{2}\right)<\infty$ resulting in

$$
\sup _{T \geq 0} \frac{1}{T} E\left(\int_{0}^{T} e^{-\alpha t} X_{0} h(t) d t\right)<\infty
$$

and $\sup _{T \geq 0} \frac{1}{T} \int_{0}^{T} h(t)^{2} d t<\infty$.

Proposition 6.9. As $T \rightarrow \infty$, we have

$$
\lim _{T \rightarrow \infty} \frac{1}{T} S_{T}=0, \text { almost surely. }
$$

Proof. Observe that $S_{T}$ is a martingale since the Lévy process is a martingale due to condition (17). By Lemma 6.8, $\frac{1}{\sqrt{T}} S_{T}$ is $L^{2}$-bounded. Doob's maximal inequality for timediscontinuous submartingales, see Theorem 2.1.5 in [1] (p. 74), provides for any $\epsilon>0$ that

$$
\begin{aligned}
P\left(\sup _{2^{k} \leq T \leq 2^{k+1}} \frac{1}{T}\left|S_{T}\right| \geq \epsilon\right) & \leq P\left(\sup _{2^{k} \leq T \leq 2^{k+1}}\left|S_{T}\right| \geq \epsilon 2^{k}\right) \\
& \leq \frac{4}{\epsilon^{2} 2^{2 k}} E\left|S_{2^{k+1}}\right|^{2}=O\left(2^{-k}\right) .
\end{aligned}
$$

Applying the Borel-Cantelli theorem, we obtain $\lim \sup _{T \rightarrow \infty} \frac{1}{T}\left|S_{T}\right| \leq \epsilon$, almost surely, and thus we have shown that $S_{T} / T \rightarrow 0$.

Proof of Theorem 1. This follows directly from Proposition 6.7 and Proposition 6.9.

Acknowledgement. We would like to thank Jeannette Woerner, TU Dortmund, and Rogemar Mamon, University of Western Ontario, for helpful advice on change of measure for jump diffusion processes. We are further grateful to Mathias Vetter, Ruhr-Universität Bochum, for his comments that improved the manuscript.

\section{REFERENCES}

[1] D. Applebaum (2004): Lévy Processes and Stochastic Calculus. Cambridge University Press.

[2] O. E. Barndorff-Nielsen and N. Shephard (2001): Non-Gaussian Ornstein-Uhlenbeck-based models and some of their uses in financial economics. Journal of the Royal Statistical Society 63, 167-241.

[3] J. P. N. Bishwal (2008): Parameter Estimation in Stochastic Differential Equations. Springer-Verlag.

[4] P. J. Brockwell, R. A. Davis and Y. Yang (2007): Estimation for nonnegative Lévy-driven Ornstein-Uhlenbeck processes. Journal of Applied Probability 44, 977-989.

[5] T. Chan (1999): Pricing contigent claims on stocks driven by Lévy processes. The Annals of Applied Probability 9, 504-528.

[6] R. Cont and P. Tankov (2004): Financial Modelling with Jump Processes. Financial Mathematics Series, Chapman \& Hall/CRC.

[7] H. Dehling, B. Franke and T. Kott (2010): Drift estimation for a periodic mean reversion process. To appear in Statistical Inference for Stochastic Processes.

[8] A. Diop and A. F. Yode (2010): Minimum distance parameter estimation for Ornstein-Uhlenbeck processes driven by Lévy process. Statistics and Probability Letters 80, 122-127.

[9] F. R. Gantmacher (1986): Matrizentheorie. VEB Deutscher Verlag der Wissenschaften.

[10] H. Geman (2005): Commodities and Commodity Derivatives. J. Wiley. 
[11] Y. Hu and H. Long (2007): Parameter estimation for Ornstein-Uhlenbeck processes driven by $\alpha$-stable Lévy motions. Communications on Stochastic Analysis 1, 175-192.

[12] Y. Hu and H. LONG (2009): Least squares estimator for Ornstein-Uhlenbeck processes driven by $\alpha$-stable Lévy motions. Stochastic Processes and their Applications 119, 2465-2480.

[13] J. JACOD and A. N. ShiRYAev (1987): Limit Theorems for Stochastic Processes. Grundlehren der mathematischen Wissenschaft 288. Springer-Verlag.

[14] H. H. Kuo (2006): Introduction to Stochastic Integration. Springer-Verlag.

[15] Y. A. Kutoyants (1984): Parameter Estimation for Stochastic Processes. Heldermann Verlag.

[16] Y. A. Kutoyants (2004): Statistical Inference for Ergodic Diffusion Processes. Springer-Verlag.

[17] R. S. Lipster and A. N. Shiryayev (1977): Statistics of Random Processes I. Springer-Verlag.

[18] P. Protter (2004): Stochastic Integration and Differential Equations. Springer-Verlag.

[19] M. Sørensen (1989): A note on the existence of a consistent maximum likelihood estimator for diffusions with jumps. Markov Processes and Control Theory, 229-234. Akademie-Verlag.

[20] M. SøRensen (1991): Likelihood methods for diffusions with jumps. Statistical Inference in Stochastic Processes, 67-105. Marcel Dekker, Inc.

[21] K. Spiliopoulos (2009): Method of moments estimation of Ornstein-Uhlenbeck processes driven by general Lévy process. Publications de L'Institut de Statistique de l'Université de Paris 53, 3-17.

Fakultät für Mathematik, Ruhr-Universität Bochum, 44780 Bochum, Germany

E-mail address: brice.franke@ruhr-uni-bochum.de

E-mail address: thomas.kott@ruhr-uni-bochum.de 


